PROCEEDINGS OF THE

AMERICAN MATHEMATICAL SOCIETY

Volume 137, Number 9, September 2009, Pages 2959-2968

S 0002-9939(09)09896-7

Article electronically published on May 6, 2009

\title{
LACUNARITY OF CERTAIN PARTITION-THEORETIC GENERATING FUNCTIONS
}

\author{
EMILY CLADER, YVONNE KEMPER, AND MATT WAGE
}

(Communicated by Jim Haglund)

\begin{abstract}
We consider a certain family of infinite products, denoted $f_{a, b}$, which were introduced by Han as a generalization of the Nekrasov-Okounkov formula. Extending the work of Serre on powers of Dedekind's $\eta$-function, we investigate the integers $a$ and $b$ for which "almost all" of the Fourier coefficients of $f_{a, b}$ are zero (forms with this property are referred to as lacunary). We give the complete list of pairs $(a, b)$, where $b$ is odd, for which $f_{a, b}$ is lacunary.
\end{abstract}

\section{INTRODUCTION AND STATEMENT OF RESULtS}

In the theory of partitions, generating functions are a prevalent and useful tool. Perhaps the most famous of these is the generating function for the partition function $p(n)$ :

$$
\sum_{n=0}^{\infty} p(n) q^{n}=\prod_{n=1}^{\infty} \frac{1}{1-q^{n}} .
$$

The subject is also home to a number of beautiful identities, such as the following, due respectively to Euler, Jacobi, and Jacobi:

$$
\begin{gathered}
\prod_{n=1}^{\infty}\left(1-q^{n}\right)=\sum_{k \in \mathbb{Z}}(-1)^{k} q^{\left(3 k^{2}+k\right) / 2}, \\
\prod_{n=1}^{\infty}\left(1-q^{n}\right)^{3}=\sum_{k \in \mathbb{Z}}(-1)^{k}(2 k+1) q^{\left(k^{2}+k\right) / 2}, \\
\prod_{n=1}^{\infty} \frac{\left(1-q^{n}\right)^{2}}{1-q^{2 n}}=\sum_{k \in \mathbb{Z}}(-1)^{k} q^{k^{2}} .
\end{gathered}
$$

While these identities can be stated combinatorially, they have partition-theoretic interpretations. All are related to modular forms, thanks to the fact that Dedekind's $\eta$-function,

$$
\eta(z)=q^{\frac{1}{24}} \prod_{n=1}^{\infty}\left(1-q^{n}\right)
$$

(where $q=e^{2 \pi i z}, \operatorname{Im}(z)>0$ ), is a weight- $\frac{1}{2}$ modular form.

Received by the editors July 31, 2008, and, in revised form, January 19, 2009.

2000 Mathematics Subject Classification. Primary 11F30, 11P82, 11F11; Secondary 11F20.

(C)2009 American Mathematical Society

Reverts to public domain 28 years from publication 
A series $\sum_{n=0}^{\infty} a(n) q^{n}$ is said to be lacunary if "almost all" of its coefficients are zero, that is, if

$$
\lim _{x \rightarrow \infty} \frac{\#\{0 \leq n<x: a(n)=0\}}{x}=1 .
$$

We note, in particular, that the series appearing in the identities (1.1), (1.2), and (1.3) are all lacunary.

In light of examples such as these, a natural question arises: for which exponents $r$ is it the case that

$$
f_{r}(z)=\prod_{n=1}^{\infty}\left(1-q^{n}\right)^{r}=\sum_{n=0}^{\infty} \tau_{r}(n) q^{n}
$$

is lacunary? In [10], Serre proved that, given a positive even integer $r$, the series $f_{r}(z)$ is lacunary if and only if $r \in\{2,4,6,8,10,14,26\}$.

Remark. The situation is not as well-understood when $r$ is odd, since in this case, $\eta^{r}$ has half-integral weight. Although it is clear from (1.1) and (1.2) that $f_{1}$ and $f_{3}$ are lacunary, little is known beyond this for odd $r \geq 5$. The best result in this direction is due to Ono [7], who proved lower bounds for the number of non-vanishing coefficients of the Fourier expansions of half-integral weight modular forms. In the case of powers of $\eta$, his result implies for odd $r \geq 5$ that

$$
\#\left\{n<x: \tau_{r}(n) \neq 0\right\} \gg_{r} x / \log x .
$$

A combinatorial interpretation is useful in motivating the investigation of the lacunarity of these series. Toward this end, we recall a fundamental result of Macdonald, in which it is demonstrated that the products $\eta^{t^{2}-1}(z)$ can in fact be viewed as combinatorial generating functions arising from the theory of infinite-dimensional Lie algebras. For odd positive integers $t$, he showed that

$$
\eta^{t^{2}-1}(z)=c_{0} \sum_{\left(v_{0}, \ldots, v_{t-1}\right)} \prod_{i<j}\left(v_{i}-v_{j}\right) q^{\left(v_{0}^{2}+v_{1}^{2}+\cdots+v_{t-1}^{2}\right) /(2 t)}
$$

Here, $c_{0}$ is an absolute numerical constant, and the sum ranges over all $V$-codings, that is, vectors of integers $\left(v_{0}, v_{1}, \ldots, v_{t-1}\right)$ such that $v_{i} \equiv i(\bmod t)$ for each $i$ and $v_{0}+\cdots+v_{t-1}=0$.

Recall that, given a Ferrers diagram associated to a partition, each square $u$ can be labeled with a hooklength, an integer that counts the number of squares $v$ such that either $v$ is in the same row as $u$ and to the right, $v$ is in the same column as $u$ and below, or $v=u$. For example, we have:

\begin{tabular}{|l|l|l|l|l|}
\hline 7 & 6 & 4 & 2 & 1 \\
\cline { 1 - 3 } 4 & 3 & 1 & \multicolumn{2}{|c}{} \\
\cline { 1 - 2 } 2 & 1 & \multicolumn{3}{|c}{} \\
\cline { 1 - 2 } & & \multicolumn{1}{|c}{}
\end{tabular}

FigurE 1. The Ferrers diagram of $\lambda=5+3+2$ with hooklengths.

Recently, using notions such as these, Nekrasov and Okounkov generalized the result of Macdonald to include all powers of $\eta$ and reformulated his identity in a 
partition-theoretic way. They showed that for any complex number $b$ we have

$$
\sum_{\lambda \in \mathcal{P}} q^{|\lambda|} \prod_{h \in \mathcal{H}(\lambda)}\left(1-\frac{b}{h^{2}}\right)=\prod_{n \geq 1}\left(1-q^{n}\right)^{b-1},
$$

where $\mathcal{P}$ denotes the set of all partitions, $\mathcal{H}(\lambda)$ denotes the multiset of hooklengths of a partition $\lambda$, and $|\lambda|$ denotes the size of $\lambda$.

From this perspective, then, Serre's theorem can be viewed as a characterization of the situations in which the partitions of an integer $n$ exhibit dramatic cancellation after being weighted in a particular combinatorial way. This strikingly resembles Franklin's proof of Euler's identity (1.1), which utilizes the observation that for all integers $n$ not expressible as $\frac{3 k^{2}+k}{2}$, the number of partitions of $n$ into an even number of distinct parts equals the number of partitions of $n$ into an odd number of distinct parts.

A partition $\lambda$ is said to be a $t$-cor 11 if $\mathcal{H}(\lambda)$ does not contain the integer $t$. These objects have a variety of implications in number theory; for example, Garvan, Kim and Stanton demonstrate in 3] the relevance of $t$-cores to certain combinatorial proofs of the famous Ramanujan congruences. In an important paper by Han [4], the Nekrasov-Okounkov formula was generalized to incorporate the notion of $t$-cores. For $a \in \mathbb{Z}^{+}$, Han showed (Theorem 1.3, $y=1$ case) that

$$
\sum_{\lambda \in \mathcal{P}} q^{|\lambda|} \prod_{h \in \mathcal{H}_{a}(\lambda)}\left(1-\frac{a b}{h^{2}}\right)=\prod_{n \geq 1} \frac{\left(1-q^{a n}\right)^{b}}{\left(1-q^{n}\right)},
$$

where $\mathcal{H}_{a}(\lambda)$ denotes the multiset of hooklengths of $\lambda$ that are multiples of $a$. When $a=1$, the identity of Nekrasov and Okounkov is recovered.

In view of Serre's work, combined with Han's partition-theoretic interpretation, we prove the following theorem:

Theorem 1.1. Define

$$
f_{a, b}(z):=q^{\frac{a b-1}{24}} \prod_{n \geq 1} \frac{\left(1-q^{a n}\right)^{b}}{\left(1-q^{n}\right)} .
$$

The complete list of pairs $(a, b)$, where $a$ and $b$ are integers with $b$ odd, for which $f_{a, b}(z)$ is lacunary is given in Table 1 below.

TABle 1. Pairs $(a, b)$ with $b$ odd for which $f_{a, b}$ is lacunary

\begin{tabular}{|c|c|}
\hline$a$ & $b$ such that $f_{a, b}$ is lacunary \\
\hline 1 & $\{3,5,7,9,11,15,27\}$ \\
2 & $\{3,5,7\}$ \\
3 & $\{3,5,9\}$ \\
4 & $\{5,7\}$ \\
5 & $\{7,11\}$ \\
6 & $\varnothing$ \\
7 & $\{9,15\}$ \\
\hline
\end{tabular}

\footnotetext{
${ }^{1}$ The condition we give is equivalent to the assertion that none of the hooklengths are multiples of $t$, which is therefore often used as the definition of a $t$-core.
} 
Remark. When $a=1$, the theorem is Serre's result on powers of $\eta$.

This result depends critically on the theory of modular forms. In particular, our arguments are inspired by those utilized by Serre in [10], wherein lacunary forms are identified with modular forms with complex multiplication (briefly, "CM"). Strictly speaking, the generalization of Serre's argument implies that for each $a$ there are at most finitely many odd $b$ such that $f_{a, b}$ is lacunary (see Lemma 3.2 below). This, however, does not preclude the possibility that there are infinitely many lacunary $f_{a, b}$. To obtain the stronger result, we make use of the Pólya-Vinogradov Inequality for short character sums.

In Section 2, we prove the modularity properties of $f_{a, b}$ using Dedekind's $\eta$ function, and we recall the notions from the theory of modular forms with complex multiplication that will be necessary for the proof of Theorem 1.1. In Section 3, we give the proof of the theorem. Finally, in Section 4, we demonstrate an explicit representation of several of the forms listed in Table 1 as a linear combination of CM forms.

\section{MOdular FORMS}

2.1. Dedekind's $\eta$-function and $f_{a, b}(z)$. Recall that Dedekind's $\eta$-function is defined by the infinite product

$$
\eta(z)=q^{\frac{1}{24}} \prod_{n \geq 1}\left(1-q^{n}\right),
$$

where $q=e^{2 \pi i z}$ and $\operatorname{Im}(z)>0$. We know (see, for example, [1) the transformation properties of $\eta$ under the two generators of the full modular group,

$$
\begin{aligned}
& \eta(z+1)=e^{\pi i / 12} \eta(z), \\
& \eta(-1 / z)=(-i z)^{1 / 2} \eta(z),
\end{aligned}
$$

from which one can deduce (see, for example, [8]) that $\eta(24 z)$ is a modular form of weight $\frac{1}{2}$ over $\Gamma_{0}(576)$, with Nebentypus character

$$
\chi_{12}(n):=\left(\frac{12}{n}\right) .
$$

Equipped with the above facts about $\eta(z)$, one can compute the transformation properties of any function of the form

$$
\prod_{\delta \mid N} \eta(\delta z)^{r_{\delta}} .
$$

Such functions are referred to as eta-quotients.

Let $a \in \mathbb{Z}^{+}, b \in \mathbb{Z}$, and define $f_{a, b}(z)$ as in (1.5). We state the conditions on $a$ and $b$ under which, after a possible change of variable, this function is a meromorphic, holomorphic, or cuspidal modular form. The proof of this result is omitted, as the criteria required to check its validity appear, for example, in Theorem 1.64 of 8 .

Lemma 2.1. Let $f_{a, b}(z)$ be as in (1.5). Then $f_{a, b}(24 z)$ is a weakly holomorphic modular form of weight $\frac{b-1}{2}$ over $\Gamma_{0}(576 a)$, with Nebentypus character

$$
\chi_{f}(d)= \begin{cases}\left(\frac{(-1)^{\frac{b-1}{2}} a}{d}\right) & \text { if } b \text { is odd, } \\ \left(\frac{3}{d}\right) & \text { if } b \text { is even. }\end{cases}
$$


Furthermore, $f_{a, b}(z)$ is holomorphic when $b \geq a$ and cuspidal when $b>a$.

Remark. Notice that in the above lemma, the level $576 a$ is not necessarily optimal. In general, all that is required is that the level is $(m n) a$ for positive integers $m$ and $n$ such that $m(a b-1) \equiv 0(\bmod 24)$ and $n(b-a) \equiv 0(\bmod 24)($ see $[8$, Theorem 1.64).

2.2. Modular forms with CM. Let $f$ be a weakly holomorphic modular form of integral weight at least two. If $f$ has a pole at one of the cusps, the HardyLittlewood circle method (see, for example [1) gives sufficient estimates for the magnitude of the coefficients of the Fourier expansion of $f$ to conclude that $f$ cannot be lacunary. Also, if $f$ is holomorphic but not cuspidal, then we can express $f$ as a linear combination of the Eisenstein series together with a cusp form; from here, the known bounds on the coefficients of such forms (for example, in [6]) similarly preclude lacunarity of $f$.

In addition, we recall that a classical result of Deligne and Serre (Proposition 9.7 of [2]) confirms that every weight-one holomorphic form on a congruence subgroup is lacunary. To prove this, they make use of exact formulas for coefficients of the Eisenstein series and their results on 2-dimensional complex Galois representations associated to weight-one modular forms.

Combining the various observations above, we see that in studying lacunarity of the forms $f_{a, b}$, we may restrict our attention to the space of cusp forms of level $N$, integer weight $k \geq 2$, and Nebentypus $\varepsilon$, which we denote by $S(N, k, \varepsilon)$. It has been proven by Serre (Theorem 17 of [9] ) that an element of $S(N, k, \varepsilon)$ is lacunary if and only if it is expressible as a linear combination of forms with $\mathrm{CM}$, so we present a characterization of such forms below.

Denote by $S_{c m}(N, k, \varepsilon)$ the subspace of $S(N, k, \varepsilon)$ generated by the forms with $\mathrm{CM}$. This space has a basis given by a collection of forms associated to Hecke characters [10:

Let $K$ be an imaginary quadratic field of discriminant $d$, and let $\varepsilon_{K}$ be the quadratic character associated to $K$; that is, $\varepsilon_{K}(p)=\left(\frac{d}{p}\right)$ for all primes $p$ not dividing $2|d|$. Let $c$ be a Hecke character on $K$ with exponent $k-1$ and conductor $f_{c}$. To $c$ we can associate a Dirichlet character $\omega_{c}$, defined by

$$
\omega_{c}(n)=c\left(n O_{K}\right) / n^{k-1} \text { for all } n \in \mathbb{Z} \text { coprime to } f_{c} .
$$

For any $\delta \in \mathbb{Z}^{+}$, we define

$$
\varphi_{K, c, \delta}(z)=\sum_{\mathfrak{a}} c(\mathfrak{a}) q^{\delta \cdot N(\mathfrak{a})},
$$

where the sum runs through ideals of $O_{K}$ coprime to $f_{c}$ and $N(\mathfrak{a})$ denotes the norm of $\mathfrak{a}$. With this definition, $\varphi_{K, c, \delta}$ is a modular form of weight $k$ and character $\varepsilon_{K} \omega_{c}$ over $\Gamma_{0}\left(\delta \cdot|d| \cdot N\left(f_{c}\right)\right)$. In order for $\varphi_{K, c, \delta}$ to be of the level, weight, and character necessary to be an element of $S(N, k, \varepsilon)$, the following two conditions are necessary and sufficient:

$$
\begin{gathered}
\delta \cdot|d| \cdot N\left(f_{c}\right) \mid N, \\
\varepsilon_{K} \omega_{c}=\varepsilon .
\end{gathered}
$$

The $\varphi_{K, c, \delta}$ satisfying (2.1) and (2.2) are exactly the generators of $S_{c m}(N, k, \varepsilon)$. 


\section{Proof of Theorem 1.1}

In this section, we provide the proof of Theorem 1.1. Recall, first, that given a modular form $f(z)=\sum a(n) q^{n}$ of integer weight $k$, level $N$, and Nebentypus character $\chi$, the action of the Hecke operator $T_{m}$ on $f$ is defined by

$$
f(z) \mid T_{m}=\sum_{n=0}^{\infty}\left(\sum_{d \mid \operatorname{gcd}(m, n)} \chi(d) d^{k-1} a\left(m n / d^{2}\right)\right) q^{n},
$$

where $\chi(n)=0$ if $\operatorname{gcd}(N, n) \neq 1$. When $m=p$ is a prime, this definition reduces to

$$
f(z) \mid T_{p}=\sum_{n=0}^{\infty}\left(a(p n)+\chi(p) p^{k-1} a(n / p)\right) q^{n} .
$$

(When $p$ does not divide $n$, we define $a(n / p)$ to be zero.)

We begin with a preliminary lemma. Its proof is well-known and similar to that of Lemma 1 of [10], so it will be omitted here. It should be noted, however, that the proof utilizes the fact that the forms $\varphi_{k, c, \delta}$ are eigenforms of all Hecke operators $T_{n}$ such that $\operatorname{gcd}(N, n)=1$. The statement is as follows:

Lemma 3.1. Let $g \in S_{c m}(N, k, \varepsilon)$. If $n$ is an integer such that $\operatorname{gcd}(N, n)=1$ and there is no ideal of norm $n$ in $\mathbb{Q}(\sqrt{-d})$ for any $d>0$ with $d \mid N$, then $g \mid T_{n}=0$.

This result can be used to show that for a fixed $a$, the odd $b$ for which $f_{a, b}$ is potentially lacunary represent the zeros of a finite collection of polynomials and are therefore a finite set.

Lemma 3.2. For each $a \in \mathbb{Z}^{+}$, there are at most finitely many odd $b$ for which $f_{a, b}$ is lacunary.

Proof. Let $a \in \mathbb{Z}^{+}$be fixed, and choose $n$ as in Lemma 3.1 We claim that there are at most finitely many odd values of $b$ such that $f_{a, b}(24 z) \mid T_{n}=0$. The above lemma shows that these are the only possible candidates for lacunarity, thereby proving the claim.

We assume, for the sake of simplicity, that $n=p$ is a prime; the proof is essentially the same in the general case. Let the coefficients $A_{a, b}$ be defined by the expansion

$$
f_{a, b}(24 z)=q^{a b-1} \prod_{n \geq 1} \frac{\left(1-q^{24 a n}\right)^{b}}{1-q^{24 n}}=\sum_{n \geq 0} A_{a, b}(n) q^{24 n+a b-1} .
$$

Then

$$
f_{a, b}(24 z) \mid T_{p}=\sum_{\substack{n \geq 0 \\ p \mid 24 n+a b-1}} A_{a, b}(n) q^{\frac{24 n+a b-1}{p}}+\chi_{f}(p) p^{\frac{b-3}{2}} \sum_{n \geq 0} A_{a, b}(n) q^{p(24 n+a b-1)} .
$$

Let $n_{0}$ be the smallest $n$ such that $p \mid 24 n+a b-1$. Then it must be the case that $0 \leq n_{0} \leq p-1$. By construction, $\frac{24 n_{0}+a b-1}{p}$ is the smallest exponent appearing in the first summation above. Furthermore, all of the terms after the first term in the second summation have exponent at least $p(24+a b-1) \geq 24+\frac{a b-1}{p}>\frac{24 n_{0}+a b-1}{p}$. Also, one easily checks that for $a \geq 2$ it is not possible to have $\frac{24 n_{0}+a b-1}{p}=p(a b-1)$, so we conclude that the exponent $\frac{24 n_{0}+a b-1}{p}$ appears nowhere else in the Fourier 
expansion of $f_{a, b}(24 z) \mid T_{p}$. Thus, to show that $f_{a, b}(24 z) \mid T_{p} \neq 0$ it is sufficient to verify that $A_{a, b}\left(n_{0}\right) \neq 0$.

Notice that

$$
\prod_{n \geq 1} \frac{\left(1-q^{24 a n}\right)^{b}}{1-q^{24 n}}=\prod_{n \geq 1}\left(1+q^{24 n}+\cdots+q^{24 n(a-1)}\right)\left(1-q^{24 a n}\right)^{b-1} .
$$

We observe that for fixed $a$ and $n$, the coefficients $A_{a, b}(n)$ may be viewed as polynomials in $b$; moreover, the degree of $A_{a, b}(n)$ is bounded by $\left\lfloor\frac{n}{a}\right\rfloor$. To motivate these observations, we exhibit the construction of $A_{2, b}(4)$. Considering the possible ways that terms from the product in (3.2) could multiply to $q^{96}$, we see that

$$
\begin{aligned}
A_{2, b}(4) & =-\left(\begin{array}{c}
b-1 \\
1
\end{array}\right)+\left(\begin{array}{c}
b-1 \\
2
\end{array}\right)-\left(\begin{array}{c}
b-1 \\
1
\end{array}\right)+1+1 \\
& =\frac{b^{2}-7 b+10}{2} .
\end{aligned}
$$

When $b=1$, none of these coefficients are zero; that is, none of the polynomials $A_{a, b}(n)$ for a given $n$ has a root at $b=1$. Therefore, we conclude that none of the polynomials are identically zero, so each has at most finitely many roots. This proves that there are at most finitely many $b$ such that $A_{a, b}\left(n_{0}\right)=0$, and hence such that $f_{a, b}(24 z) \mid T_{p}=0$, so the lemma is proved.

To complete the proof of Theorem 1.1. we will use the preceding lemmas in conjunction with the Pólya-Vinogradov Inequality, which implies that, given a nontrivial Dirichlet character $\chi$ with modulus $m$, the following holds:

$$
\left|\sum_{x=1}^{h} \chi(x)\right| \leq 2 \sqrt{m} \log (m) .
$$

Proof of Theorem 1.1. Let $a$ be fixed and let $a^{\prime}$ be the largest square-free divisor of $6 a$, that is, the square-free part of the level of $f_{a, b}$. Define $\psi_{p}(n)=\left(\frac{-p}{n}\right)$.

Recall that to satisfy the conditions of Lemma 3.1, we must choose an integer $n$ that is relatively prime to the level of $f_{a, b}$ and such that there does not exist an ideal of norm $n$ in $\mathbb{Q}(\sqrt{-d})$ for any $d$ dividing the level of $f_{a, b}$. It suffices to assume that $\psi_{p}(n)=\left(\frac{-p}{n}\right)=-1$ for all primes $p \mid a^{\prime}$ and $\left(\frac{-1}{n}\right)=-1$. For indeed, these conditions imply that $\left(\frac{-d}{n}\right)=-1$ for all positive integers $d$ dividing the level of $f_{a, b}$. By considering the prime factorization of $n$ together with multiplicativity in the denominator of the Kronecker symbol and using the characterization of the splitting of primes in imaginary quadratic fields in terms of the Legendre symbol, one sees that this, in turn, implies the requisite conditions.

Assume without loss of generality that $a^{\prime}=6 a$. Then it suffices to find an $n \equiv 23(\bmod 24)$ such that $\psi_{p}(n)=-1$ for all primes $p \mid a$. Let $a=p_{1} p_{2} \cdots p_{m}$, where the $p_{i}$ are distinct primes. Consider the following linear combination of Dirichlet characters:

$$
g_{a}(n):=\frac{\sum_{d \mid a} \mu(d) \psi_{d}(n)}{2^{m}},
$$

where $\psi_{d}(n):=\prod_{p \mid d} \psi_{p}(n)$. It can be shown that

$$
g_{a}(n)= \begin{cases}1 & \text { if }\left(\frac{-p}{n}\right)=-1 \text { for all } p \mid a \\ 0 & \text { otherwise }\end{cases}
$$


To verify that this representation of $g_{a}(n)$ is equivalent to the above definition, one can induct on the number of prime divisors of $a$, noticing that if $q$ is a prime not dividing $a$, then $g_{a \cdot q}(n)=\frac{\left(1-\psi_{q}(n)\right) g_{a}(n)}{2}$.

Therefore, the desired $n$ will satisfy $g_{a}(n)=1$ and $n \equiv 23(\bmod 24)$. We will use the Pólya-Vinogradov Inequality to show that for sufficiently large $a$,

$$
\sum_{\substack{n<a \\ n \equiv 23(24)}} \sum_{d \mid a} \mu(d) \psi_{d}(n)>0 .
$$

This implies that there exists an $n$ meeting the above conditions with $n<a$. By the proof of Lemma 3.2. the set of odd $b$ for which $f_{a, b}$ is lacunary is a subset of the roots of the polynomials $A_{a, b}(0), \ldots, A_{a, b}(n-1)$. If $n<a$, these will all be constant, so there will be no such $b$.

To prove (3.4), let $S$ be the set of Dirichlet characters modulo 24. Then

$$
\begin{aligned}
\sum_{\substack{n<a \\
n \equiv 23(24)}} \sum_{d \mid a} \mu(d) \psi_{d}(n) & =\sum_{\substack{n<a \\
n \equiv 23(24)}} 1+\sum_{\substack{d \mid a \\
d>1}} \mu(d) \sum_{\substack{n<a \\
n \equiv 23(24)}} \psi_{d}(n) \\
& =\sum_{\substack{n<a \\
n \equiv 23(24)}} 1+\sum_{\substack{d \mid a \\
d>1}} \mu(d) \sum_{n<a} \psi_{d}(n) \sum_{\chi \in S} \frac{\chi(-n)}{8} \\
& \geq \frac{a}{24}-1+\sum_{\substack{d \mid a \\
d>1}} \mu(d) \frac{1}{8} \psi_{d}(-1) \sum_{\chi \in S} \sum_{n<a} \psi_{d}(-n) \chi(-n) .
\end{aligned}
$$

This follows from the fact that $\sum_{\chi \in S} \chi(1)=8$.

Applying (3.3) to the innermost sum above, combined with the fact that $a>d$ for all $d \mid a$, we have

$$
\begin{aligned}
\sum_{\substack{n<a \\
n \equiv 23(24)}} \sum_{d \mid a} \mu(d) \psi_{d}(n) & \geq \frac{a}{24}-1-\sum_{\substack{d \mid a \\
d>1}} 2 \sqrt{24 d} \log (24 d) \\
& \geq \frac{a}{24}-1-2^{m+1} \sqrt{24 a} \log (24 a) .
\end{aligned}
$$

If $m \geq 12$, then $\frac{a}{24}-1-2^{m+1} \sqrt{24 a} \log (24 a)>0$ for all $a$, so there are no corresponding odd $b$ for any $a$ with at least 12 prime divisors. For each $m<12$, there are at most finitely many $a$ such that inequality (3.4) does not hold. These are the only $a$ for which there may exist odd $b$ where $f_{a, b}$ is lacunary, and each yields at most finitely many such $b$. Therefore, the complete list of pairs $(a, b)$ where $b$ is odd and $f_{a, b}$ is lacunary is finite. Verifying all possible cases by machine computation, we find that the precise list is given in Table 1.

\section{EXAMPLeS}

In this section, we explain the computation utilized to verify that each of the forms $f_{a, b}$ in Table 1 (recalled for reference below) is lacunary, and we give an explicit representation as a linear combination of CM forms in certain cases.

For all cases, we use the fact that for a given choice of $a$ and $n$, the coefficient $A_{a, b}(n)$ on $q^{n}$ is visibly a polynomial in $b$ of degree at most $\left\lfloor\frac{n}{a}\right\rfloor$. By computing the value of this polynomial for $b \in\left\{1, \ldots,\left\lfloor\frac{n}{a}\right\rfloor+1\right\}$ and using Lagrange interpolation, 


\begin{tabular}{|c|c|}
\hline$a$ & $b$ such that $f_{a, b}$ is lacunary \\
\hline 1 & $\{3,5,7,9,11,15,27\}$ \\
2 & $\{3,5,7\}$ \\
3 & $\{3,5,9\}$ \\
4 & $\{5,7\}$ \\
5 & $\{7,11\}$ \\
6 & $\varnothing$ \\
7 & $\{9,15\}$ \\
\hline
\end{tabular}

we can obtain the polynomial explicitly. The set consisting of the roots of the polynomials $A_{a, b}(0), \ldots, A_{a, b}(p-1)$, where $p$ is inert in all fields with discriminant dividing $576 a$, contains all the possible $b$ such that $f_{a, b}$ is lacunary.

Some of these values of $b$, however, can be immediately discarded. Recall from the proof of Lemma 3.2 that $f_{a, b}$ is not a candidate for lacunarity unless $A_{a, b}\left(n_{0}\right)=$ 0 , where $n_{0}$ is the smallest $n$ such that $p \mid 24 n+a b-1$. In particular, it must be the case that $24 n_{0} \equiv 1-a b(\bmod p)$, so those roots $b$ not satisfying this condition need not be considered.

From among the remaining possible values of $b$, we can verify lacunarity by displaying a representation of $f_{a, b}$ as a linear combination of CM forms, as is demonstrated below.

Example $(a=2, b=3)$. We express $f_{a, b}$ as a linear combination of Hecke eigenforms as follows:

$$
f_{2,3}=\frac{1}{4 i}\left(x_{2,3}-y_{2,3}\right)
$$

where

$$
x_{2,3}=2 i f_{a, b}+f_{a, b} \mid T_{5}=q+2 i q^{5}-3 q^{25}+2 i q^{29}+q^{49}+\ldots
$$

and $y_{2,3}=\overline{x_{2,3}}$. Both $x_{2,3}$ and $y_{2,3}$ have complex multiplication by $\mathbb{Q}(\sqrt{-1})$. This can be verified by subtracting each from its twist by the quadratic character

$$
\chi(p)=\left\{\begin{aligned}
1 & \text { if } p \equiv 1(\bmod 4) \\
-1 & \text { if } p \equiv 3(\bmod 4),
\end{aligned}\right.
$$

which yields a Fourier expansion that is identically zero.

Example $(a=4, b=7)$. We express $f_{a, b}$ as a linear combination of Hecke eigenforms as follows:

$$
f_{4,7}=\frac{-1}{56}\left(x_{4,7}+y_{4,7}+z_{4,7}+w_{4,7}\right),
$$

where

$$
\begin{gathered}
x_{4,7}=2 f_{4,7}-4\left(f_{4,7} \mid T_{5}\right)+f_{4,7} \mid T_{17}=q^{3}-8 q^{15}+9 q^{27}+24 q^{39}+\ldots, \\
z_{4,7}=-30 f_{4,7}+\frac{4}{3} i \sqrt{2}\left(f_{4,7} \mid T_{11}\right)+f_{4,7} \mid T_{17}=q^{3}+4 i \sqrt{2} q^{9}-23 q^{27}+\ldots
\end{gathered}
$$

and $y_{4,7}=\overline{x_{4,7}}, w_{4,7}=\overline{z_{4,7}}$. The forms $x_{4,7}$ and $y_{4,7}$ have complex multiplication by $\mathbb{Q}(\sqrt{-1})$, while $z_{4,7}$ and $w_{4,7}$ have complex multiplication by $\mathbb{Q}(\sqrt{-2})$, as can be checked by methods analogous to the above. 


\section{ACKNOWLEDGEMENTS}

The authors wish to thank Professor Ken Ono for his guidance and suggestions. They also thank Frank Thorne, Amanda Folsom, and Rob Rhoades for their many helpful comments. Finally, they thank the National Science Foundation, the Manasse family, and the Hilldale Foundation for supporting this work.

\section{REFERENCES}

[1] T. Apostol, Modular Functions and Dirichlet Series in Number Theory, Graduate Texts in Mathematics, Second Edition, Springer-Verlag, New York, 1997. MR1027834 (90j:11001)

[2] P. Deligne and J.-P. Serre, Formes modulaires de poids 1, Ann. Sci. Éc. Norm. Sup. 7 (1974), pp. 507-530. MR0379379 (52:284)

[3] F. Garvan, D. Kim, D. Stanton, Cranks and t-cores, Invent. Math. 101 (1990), pp. 1-17. MR:1055707 (91h:11106)

[4] G-N. Han, The Nekrasov-Okounkov hook length formula: refinement, elementary proof, extension, and applications (preprint).

[5] H. Iwaniec, Topics in Classical Automorphic Forms, Graduate Studies in Mathematics, Vol. 17, American Mathematical Society, Providence, RI, 1997. MR.1474964 (98e:11051)

[6] N. Koblitz, Introduction to Elliptic Curves and Modular Forms, Graduate Texts in Mathematics, Second Edition, Springer-Verlag, New York, 1993. MR.1216136 (94a:11078)

[7] K. Ono, Gordon's E-conjecture on the lacunarity of modular forms, Comptes Rendus Math. Rep. of Acad. Sci. Canada 20, No. 4 (1998), pp. 103-107. MR1662100 (99m:11055)

[8] K. Ono, The Web of Modularity: Arithmetic of the Coefficients of Modular Forms and qseries, CMBS Regional Conference Series in Mathematics, American Mathematical Society, Providence, RI, 2004. MR2020489 (2005c:11053)

[9] J-P. Serre, Quelques applications du théorème de densité de Chebatorev, Publ. Math. I.H.E.S. 54 (1981), pp. 123-201.

[10] J-P. Serre, Sur la lacunarité des puissances de $\eta$, Glasgow Math. J. 27 (1985), pp. 203-221. MR819840(87e:11066)

Department of Mathematics, Columbia University, New York, New York 10027

Department of Mathematics, University of California Berkeley, Berkeley, CaliforNIA 94708

Appleton East High School, 1411 N. Briarcliff Drive, Appleton, Wisconsin 54915

Current address: Princeton University, 0920 Frist Campus Center, Princeton, New Jersey 08544 